\title{
Risk stratification in diabetic patients: A continuing challenge
}

\author{
Todd D. Miller, MD, a and Leslee J. Shaw, PhD $^{\mathrm{b}}$
}

\section{See related article, pp. 524-532}

Our current paradigm for treating coronary artery disease (CAD) focuses on improving symptom status and correctly risk stratifying patients. Patients with severe symptoms, especially those with symptoms refractory to medical therapy, are candidates for percutaneous coronary intervention (PCI) or coronary artery bypass grafting (CABG) to enhance quality of life. This issue generally does not apply to patients who are asymptomatic or have only mild symptoms (acknowledging that some patients who are mildly symptomatic will become asymptomatic following successful PCI or $\mathrm{CABG}$ ). In these patients, the major issue to address is accurate risk stratification. The identification of any CAD can determine the intensity of treatment of risk factors, and the identification of severe (left main or 3-vessel) CAD can lead to consideration of CABG for improving survival.

Selected subsets of patients who can be identified as those with higher risk on the basis of simple clinical characteristics theoretically might benefit from more aggressive risk stratification. Patients with diabetes represent such a higher-risk patient subset. Some form of heart disease or stroke accounts for $65 \%$ of deaths in

From the Division of Cardiovascular Diseases, ${ }^{\text {a }}$ Department of Medicine, Mayo Clinic, Rochester, MN and Division of Cardiology, Department of Medicine, Emory University School of Medicine, Atlanta, GA.

Disclosures for Dr. Todd Miller: I receive research grants from Lantheus Medical Imaging and Molecular Insight Pharmaceuticals and have consulting agreements with TherOx, Inc. and The Medicines Company.

Disclosures for Dr. Leslee J. Shaw: I receive research grants from GE Healthcare and Bracco Diagnostics, Inc.

Received for publication Feb 11, 2009; final revision accepted Feb 11, 2009.

Reprint requests: Todd D. Miller, MD, Division of Cardiovascular Diseases, Department of Medicine, Mayo Clinic, Gonda 5, 200 First Street SW, Rochester, MN 55905; miller.todd@mayo.edu.

J Nucl Cardiol 2009;16:486-9.

$1071-3581 / \$ 34.00$

Copyright (c) 2009 by the American Society of Nuclear Cardiology. doi:10.1007/s12350-009-9070-4 diabetic patients. ${ }^{1}$ The CAD mortality rates in diabetics without clinically evident CAD have been reported to be as high as mortality rates in non-diabetics with established $\mathrm{CAD},{ }^{2,3}$ leading to the designation of diabetes as a "CAD risk equivalent." 4 In 2000, there were an estimated 171 million patients with diabetes worldwide, and this number is projected to increase to 366 million by the year $2030 .^{5}$ Performing accurate risk stratification in asymptomatic or mildly symptomatic diabetic patients is an important health priority. 6,7

Several studies applying a retrospective approach have examined the value of screening stress single photon emission computed tomography myocardial perfusion imaging (SPECT MPI) in primarily asymptomatic diabetic patients. ${ }^{8-13}$ These studies reported a high yield of abnormal tests, ranging between $25 \%$ and $50 \%$. SPECT MPI has also been shown to risk stratify these populations. However, a concern that has emerged from stress imaging studies in patients with diabetes (symptomatic or asymptomatic) is the inability to identify a true low-risk population. ${ }^{14}$ Annual cardiac death or myocardial infarction (MI) rates in retrospective studies of diabetic patients with normal or low-risk SPECT MPI scans were in the range of $2-3 \%$, higher than the average annual hard event rate of $0.6 \%$ reported in general patient populations with normal or low-risk images. $^{15}$

The Detection of Ischemia in Diabetics (DIAD) study represents the only prospective study designed to examine the nonselective application of stress SPECT MPI in asymptomatic diabetic patients. ${ }^{16}$ In contrast to the retrospective studies, patients enrolled in DIAD represented a substantially "healthier' population of asymptomatic diabetic patients. Only $16 \%$ of the patients randomized to the imaging arm had abnormal SPECT MPI, including just $1 \%$ with defects $>10 \%$ of the left ventricle. The DIAD 5-year outcome has been recently published. ${ }^{17}$ The annual hard cardiac event rate was surprisingly low at $0.69 \%$. Despite the low event rate, stress SPECT MPI was able to risk stratify the population. The only other large prospective study to screen asymptomatic diabetic patients for CAD using stress SPECT MPI was performed by Anand et al. ${ }^{18}$ Their study design employed a step-wise strategy, whereby all the patients underwent CT calcium scoring followed by stress SPECT MPI in those with Agatston 
scores $>100$. The yield of abnormal scans was $32 \%$, higher than in DIAD. Although the number of events was small (11 hard events during median follow-up of 2.2 years), SPECT MPI was effective for risk stratification in this study also.

The MERIDIAN trial ${ }^{19}$ represents the first prospective study to compare the outcomes of type II diabetic patients with mild stable angina (Canadian Cardiovascular Society I-II) and reversible SPECT MPI defects randomized to medical therapy alone vs an early invasive strategy. The main MERIDIAN trial was terminated prematurely because of slow recruitment, an altogether unfortunate obstacle encountered in many randomized trials in the current era. The premature termination of the trial resulted in inadequate statistical power to test the original study hypothesis.

In this issue of the Journal, Wiersma et al. ${ }^{20}$ report the results of a sub-analysis of the MERIDIAN study. They examined the prognostic value of stress SPECT MPI in 319 patients screened for enrollment into the trial. The yield of testing was high, with perfusion defects being present in $65 \%$ of patients, including $46 \%$ with reversible defects. A breakdown of test results by the summed difference score (SDS) revealed no ischemia (SDS < 3) in 54\%, moderate ischemia (SDS 3-7) in $26 \%$, and severe ischemia (SDS $\geq 8$ ) in $20 \%$. During a mean follow-up of $2.2 \pm .6$ years, there were 14 initial hard cardiac events ( 6 deaths and 8 non-fatal myocardial infarctions). Annual event rates according to SDS categories were $0.8 \%$ for no ischemia, $1.5 \%$ for moderate ischemia, and $5.8 \%$ for severe ischemia (statistically significant differences).

The MERIDIAN study investigators are to be congratulated for publishing these data. They were unable to address their original study hypothesis, but they have assimilated the available generated data into a clinically meaningful manuscript. Although this study was performed as a post-hoc data analysis, it represents the first prospective assessment of the prognostic value of stress SPECT MPI in mildly symptomatic diabetic patients. From the standpoint of nuclear imaging, the most positive aspect of this study is that SPECT ischemia could effectively and accurately risk stratify these diabetic patients.

Additional results from this study, however, demonstrate the limited value of SPECT imaging for risk stratification in this population. The clinical model that predicted events contained two variables: insulin use and previous MI. The stronger variable was insulin use, which has been shown to be an independent prognostic variable in other studies. ${ }^{21,22}$ The global $\chi^{2}$ of the clinical model was 128.2 with a C-index of 0.74 . The addition of the variable severe ischemia ( $\operatorname{SDS} \geq 8$ ) to the clinical model resulted in a multivariable model with a significantly higher global $\chi^{2} 139.58(P<.001)$ and a moderately high C-index of 0.84 . As the authors acknowledge, this 3-variable model may have been overfitted since it was based on only 14 events and should be, therefore. considered exploratory. Although the statistically significant increase in the model $\chi^{2}$ with the addition of SPECT ischemia to the clinical variables represents a positive finding, this analysis fails to indicate how many patients were correctly reclassified by the application of SPECT MPI. Unfortunately, Wiersma and colleagues did not perform a data analysis approach known as the net reclassification index (NRI). ${ }^{23}$ The NRI can reveal how many patients are correctly reclassified by the use of a test and should be performed in prognostic studies that apply incremental statistical modeling. Clinicians can more readily appreciate changes in the number of patients who are correctly reclassified compared to the more abstract concept of changes in statistical model $\chi^{2}$ values. Given the fairly modest increases in the model $\chi^{2}$ values and C-indices and the small number of events, SPECT MPI in this study likely resulted in the correct reclassification of very few patients. More often than not, SPECT MPI results in favorable reclassification to event-free status following low risk results, and these results would likely be similar in the MERIDIAN trial.

It is interesting to compare the results of this study to the DIAD trial. The yield of testing was substantially higher in the present study (65\% perfusion defect, $20 \%$ large ischemic defect) than in DIAD (16\% perfusion defect, $1 \%$ large defect). The event rate was also higher in this study (annual death/MI approximately $2 \%$ ) than in DIAD (.69\%). There were several differences in clinical variables that identified the DIAD patients as lower risk rather than just the simple difference in symptom status (asymptomatic vs mildly symptomatic) between these two study populations. The DIAD study excluded patients with a prior history of CAD or abnormal ECG. In contrast, among patients screened for the MERIDIAN trial, 29\% had prior MI, 27\% prior PCI, $18 \%$ prior CABG, and 50\% ECG abnormalities. In addition, the MERIDIAN patients were older than those in DIAD (mean age 65 years vs 60 years) and included more males ( $63 \%$ vs $53 \%)$. The duration of diabetes was similar between the two studies ( 8 years), but more MERIDIAN patients used insulin (38\%) compared to DIAD patients $(22 \%)$.

What do these data from the MERIDIAN trial add to the published literature? Their major value is the demonstration of the accuracy of SPECT imaging for risk stratification in a prospectively identified patient population. This study along with the DIAD study ${ }^{16}$ and the study of Anand et al. ${ }^{18}$ are the only studies that have examined the prognostic value of SPECT in 
prospectively identified populations of diabetic patients. These prospective studies are not as influenced by patient selection bias, a significant limitation of studies performed using a retrospective study design. In both this study and DIAD, SPECT MPI accurately identified low-risk patients whose annual hard event rate was $<1 \%$, in contrast to the studies performed using a retrospective study design. The major limitation of these prospective studies has been the failure to identify a large enough high-risk subset of patients to justify the widespread use of SPECT for risk stratification purposes in asymptomatic or mildly symptomatic diabetic patients. Although identifying the presence of any CAD in general patient populations can be useful to gauge the aggressiveness of risk factor modification, national guidelines currently recommend more aggressive treatment of lipids ${ }^{4}$ and blood pressure ${ }^{24}$ in diabetic patients simply on the basis of diabetes status and not on whether CAD is present or absent. The major goal of noninvasive testing in diabetic patients should be to identify those with high-risk anatomical CAD. In the BARI trial, which enrolled primarily severely symptomatic diabetic patients with multivessel CAD, CABG resulted in better survival rate than PCI. ${ }^{25}$ Non-randomized data have shown that asymptomatic diabetic patients with severe perfusion defects by stress SPECT also have better survival rate when treated with CABG than with PCI or medical therapy alone. ${ }^{26} \mathrm{~A}$ test being applied primarily for risk stratification purposes should meet the following criteria: (1) the identification of a fairly large number of high-risk patients in the population being tested; (2) the use of a different treatment strategy in these high-risk patients, which they would not have otherwise received if not for the results of the test; and, (3) an improvement in outcome in the high-risk subset because of the different treatment strategy. The broad use of SPECT MPI in all asymptomatic or mildly symptomatic diabetic patients is unlikely to represent a viable approach. The ongoing challenge for future studies of SPECT MPI in these patients for risk stratification purposes will be to target an enriched subset of higher risk diabetic patients in whom this approach will be cost effective.

\section{References}

1. Lloyd-Jones D, Adams R, Carnethon M, De Simone G, Bruce Ferguson T, Flegal K, et al. Heart disease and stroke statistics 2009 Update. A report from the American Heart Association Statistics Committee and Stroke Statistics Subcommittee. Circulation 2008;119:e1-161.

2. Haffner SM, Lehto S, Ronnemaa T, Pyorala K, Laakso M. Mortality from coronary heart disease in subjects with type 2 diabetes and in nondiabetic subjects with and without prior myocardial infarction. N Engl J Med 1998;339:229-34.
3. Whiteley L, Padmanabhan S, Hole D, Isles C. Should diabetes be considered a coronary heart disease risk equivalent?: Results from 25 years of follow-up in the Renfrew and Paisley survey. Diabetes Care 2005;28:1588-93.

4. Expert Panel of Detection Evaluation and Treatment of High Blood Cholesterol in Adults. Executive summary of the third report of the National Cholesterol Education Program (NCEP) expert panel on detection, evaluation, and treatment of high blood cholesterol in adults (Adult Treatment Panel III). JAMA 2001;285:2486-97.

5. Wild S, Roglic G, Green A, Sicree R, King H. Global prevalence of diabetes. Estimates for the year 2000 and projections for 2030 . Diabetes Care 2004;27:1047-53.

6. Grundy SM, Benjamin IJ, Burke GL, Chait A, Eckel RH, Howard $\mathrm{BV}$, et al. Diabetes and cardiovascular disease. A statement for healthcare professionals from the American Heart Association. Circulation 1999;100:1134-46.

7. Bax JJ, Bonow RO, Tschope D, Inzucchi SE, Barrett E, on behalf of the Global Dialogue Group for the Evaluation of Cardiovascular Risk in Patients with Diabetes. The potential of myocardial perfusion scintigraphy for risk stratification of asymptomatic patients with type 2 diabetes. J Am Coll Cardiol 2006;48:754-60.

8. Koistinen MJ. Prevalence of asymptomatic myocardial ischemia in diabetic subjects. Brit Med J 1990;301:92-5.

9. Vanzetto G, Halimi S, Hammond T, Fagret D, Benhamon PY, Cordonnier D, et al. Prediction of cardiovascular events in clinically selected high-risk NIDDM patients: Prognostic value of exercise stress test and thallium-201 single-photon emission computed tomography. Diabetes Care 1999;22:19-26.

10. Janand-Delenne B, Savin B, Habib G, Bory M, Vague P, Lassmann-Vague V. Silent myocardial ischemia in patients with diabetes. Who to screen? Diabetes Care 1999;22:1396-400.

11. DeLorenzo A, Lima RS, Sequeira-Filho AG, Pantoja MR. Prevalence and prognostic value of perfusion defects detected by stress technetium-99m sestamibi myocardial perfusion single-proton emission computed tomography in asymptomatic patients with diabetes mellitus and no known coronary artery disease. Am J Cardiol 2002;90:827-32.

12. Zellweger MJ, Hachammovitch R, Kang X, Hayes SW, Friedman JD, Germano G, et al. Prognostic relevance of symptoms versus objective evidence of coronary artery disease in diabetic patients. Eur Heart J 2004;25:543-50.

13. Rajagopalan N, Miller TD, Hodge DO, Frye RL, Gibbons RJ. Identifying high-risk asymptomatic diabetic patients who are candidates for screening stress single-photon emission computed tomographic imaging. J Am Coll Cardiol 2005;45:43-9.

14. Kamalesh M, Feigenbaum H, Sawada S. Assessing prognosis in patients with diabetes mellitus-the Achilles' heel of cardiac stress imaging tests? Am J Cardiol 2007;99:1016-9.

15. Shaw LJ, Iskandrian AE. Prognostic value of gated myocardial perfusion SPECT. J Nuclear Cardiol 2004;11:171-85.

16. Wackers FJ, Young LH, Inzucchi SE, Chyun DA, Davey JA, Barrett EJ, et al. Detection of silent myocardial ischemia in asymptomatic diabetic subjects: The DIAD study. Diabetes Care 2004;27:1954-61.

17. Young LH, Wackers FJ, Chyun DA, Davey JA, Barrett EJ, Taillefer $\mathrm{R}$, et al. Cardiac outcomes after screening for asymptomatic coronary artery disease in patients with type 2 diabetes: The DIAD study: A randomized controlled trial. JAMA 2009;301(15):1547-55.

18. Anand DV, Lim E, Hopkins D, Corder R, Shaw LJ, Sharp P, et al. Risk stratification in uncomplicated type 2 diabetes: Prospective evaluation of the combined use of coronary artery calcium imaging and selective myocardial perfusion scintigraphy. Eur Heart $\mathbf{J}$ 2006;27:713-21. 
19. Wiersma JJ, Dijksman LE, Ten Holt WL, et al. Cardiac complications in type 2 diabetic patients with mild anginal complaints and documented reversible myocardial perfusion defects, results of the MERIDIAN trial. Neth Heart J 2006;14:409-16.

20. Wiersma JJ, Verberne H, Ten Holt WL, Radder I, Dijksman LE, Van Eck-Smit B, et al. Prognostic value of myocardial perfusion scintigraphy in type 2 diabetic patients with mild, stable angina pectoris. J Nuclear Cardiol 2008. doi: 10.1007/s12350-0099111-z.

21. Ho KT, Miller TD, Hodge DO, Bailey KR, Gibbons RJ. Use of a simple clinical score to predict prognosis of patients with normal or mildly abnormal resting electrocardiographic findings undergoing evaluation for coronary artery disease. Mayo Clin Proc 2002;77:515-21.

22. Berman DS, Kang X, Hayes SW, Friedman JD, Cohen I, Abidov A, et al. Adenosine myocardial perfusion single-photon emission computed tomography in women compared with men. Impact of diabetes mellitus on incremental prognostic value and effect on patient management. J Am Coll Cardiol 2003;41:1125-33.

23. Cook NR. Statistical evaluation of prognostic versus diagnostic model: Beyond the ROC curve. Clin Chem 2008;54:17-23.

24. National Heart Lung and Blood Institute. Seventh report of the joint national committee on prevention, detection, evaluation, and treatment of high blood pressure (JNC 7). Available at http://www.nhlbi.nih.gov/guidelines/hypertension/jnc7full.htm.

25. The BARI Investigators. Seven-year outcome in the Bypass Angioplasty Revascularization Investigation (BARI) by treatment and diabetic status. J Am Coll Cardiol 2003;35:1122-9.

26. Sorajja P, Chareonthaitawee P, Rajagopalan N, Miller TD, Frye $\mathrm{RL}$, Hodge DO, et al. Improved survival in asymptomatic diabetic patients with high-risk SPECT imaging treated with coronary artery bypass grafting. Circulation 2005;112:1311-6. 